\title{
Control químico del manchado de hojas y cálices de jamaica en Guerrero, México
}

\author{
Santo Ángel Ortega-Acosta ${ }^{1}$, Daniel Leobardo Ochoa-Martínez ${ }^{1}$, Santos Gerardo Leyva-Mir², Ciro Velasco-Cruz ${ }^{1}$, \\ José Antonio Mora-Aguilera ${ }^{1}$, Javier Hernández-Morales ${ }^{1}$
}

${ }^{1}$ Colegio de Postgraduados, Fitosanidad-Fitopatología, Carretera México-Texcoco Km. 36.5, Montecillo, Texcoco, CP. 56230, Estado de México, México. ${ }^{2}$ Universidad Autónoma Chapingo, Departamento de Parasitología Agrícola, Km 38.5 carretera México - Texcoco, Chapingo, CP. 56230, Estado de México, México

Autor para correspondência: Javier Hernández Morales (hjavier@colpos.mx)

Data de chegada: 28/06/2017. Aceito para publicação em: 26/06/2018.

$10.1590 / 0100-5405 / 182006$

\section{RESUMEN}

Ortega-Acosta, S.A.; Ochoa-Martínez, D.L.; Leyva-Mir, S.G.; Velasco-Cruz, C.; Mora-Aguilera, J.A.; Hernández-Morales, J.. Control químico del manchado de hojas y cálices de jamaica en Guerrero, México. Summa Phytopathologica, v.45, n.1, p.38-43, 2019.

El manchado de hojas y cálices de la jamaica, causado por Corynespora cassiicola, se considera la principal limitante fitosanitaria del cultivo de jamaica (Hibiscus sabdariffa) en el Estado de Guerrero, México, causando pérdidas significativas de la producción. Para su control, se evaluó la efectividad biológica de diversas substancias químicas (convencionales y alternativas) durante los ciclos de producción 2014 y 2015. El estudio se realizó en los municipios de Ayutla y Tecoanapa, Guerrero, bajo condiciones de campo y presión natural de inóculo. Las substancias químicas (tratamientos), se aplicaron a intervalos de 7 y 14 días. En cada tratamiento, se registró la severidad en hojas y cálices, así como el peso fresco y seco de cada unidad experimental. El ensayo se estableció bajo un diseño bloques al azar con cuatro repeticiones. Para el análisis de los datos se realizó un análisis de varianza y pruebas de separación de medias (Tukey-Kramer, $\alpha=0.05$ ). La menor severidad de la enfermedad y mayor peso fresco y seco de cálices se obtuvo en plantas tratadas con mancozeb, clorotalonil y benomilo alternado con oxicloruro de cobre.

Palabras clave: Corynespora cassiicola, efectividad biológica, Hibiscus sabdariffa.

\section{RESUMO}

Ortega-Acosta, S.A.; Ochoa-Martínez, D.L.; Leyva-Mir, S.G.; Velasco-Cruz, C.; Mora-Aguilera, J.A.; Hernández-Morales, J. Controle químico das folhas e cálices da jamaica em Guerrero, México. Summa Phytopathologica, v.45, n.1, p.38-43, 2019.

A mancha de folhas e cálices da jamaica, causada por Corynespora cassiicola, é a principal limitante fitossanitária da cultura da vinagreira (Hibiscus sabdariffa) no Estado de Guerrero, México, provocando perdas significativas na produção. Para seu controle, se avaliou a eficácia biológica de vários produtos químicos (convencionais e alternativos) na safra de 2014 e 2015. $\mathrm{O}$ estudo foi conduzido nos municípios de Ayutla e Tecoanapa, Guerrero, sob condições de campo e de pressão do inoculo natural. As Substâncias químicas (tratamentos) foram aplicadas em intervalos de 7 e 14 dias. Em cada tratamento, a gravidade nas folhas e cálices, bem como o peso fresco e seco para cada unidade experimental foi registrado. $\mathrm{O}$ ensaio se estabeleceu num desenho experimental de blocos causalizados com quatro repetições. Os dados foram submetidos a análise de variância e testes de separação (Tukey-Kramer, $\alpha=0.05$ ). A menor severidade da doença e o maior ganho no peso fresco e seco de cálices foi observado nos tratamentos com mancozebe, clorotalonil e benomyl alternado com oxicloreto de cobre.

Palavras-chave: Corynespora cassiicola, eficácia de controle, Hibiscus sabdariffa.

En México se destinan anualmente más de 20,000 ha para la producción de jamaica (Hibiscus sabdariffa L.), siendo el Estado de Guerrero, México, el principal productor con más del $70 \%$ de la superficie (15). De la planta se aprovechan los cálices principalmente para elaborar bebidas refrescantes y productos como té, mermeladas y licores. En el estado de Guerrero se ha presentado desde hace varios años una enfermedad denominada manchado de hojas y cálices de la jamaica inducida por Corynespora cassiicola (Berk. \& M.A. Curtis) C.T. Wei (11). Esta enfermedad tiene una amplia distribución en la zona y se considera la principal problemática fitosanitaria del cultivo, debido a las pérdidas significativas de la producción de cálices causada por este patógeno. Los niveles de incidencia pueden ser del 100\% (11). Además, en Guerrero se han estimado pérdidas totales en algunas plantaciones de jamaica, Los cálices afectados presentan problemas para ser comercializados por su mala apariencia y en casos extremos el producto es rechazado. Se ha observado que las etapas fenológicas de floración y desarrollo de cálices son las más susceptibles al daño por Corynespora cassiicola en este cultivo. Por otra parte, diversos fungicidas convencionales se han utilizado para el control de $C$. cassiicola en diferentes cultivos $(13,19)$, sin embargo, la búsqueda de métodos alternativos está tomando importancia debido a los efectos generalmente perjudiciales de dichas substancias (14). En este sentido, la aplicación de productos biorracionales como aceites esenciales, sales minerales e inductores de resistencia, entre otros, se han evaluado en los últimos años para el manejo de diversas enfermedades $(6,7,13)$ debido a que, en general, son de bajo riesgo para la salud humana y 
medio ambiente. Desafortunadamente, los productos biorracionales generalmente son más variables y menos efectivos en el manejo de enfermedades en campo comparado con los fungicidas sintéticos (6).

En México no se cuentan con opciones de manejo que permitan reducir el daño inducido por C. cassiicola en el cultivo de jamaica. Por lo anterior, el objetivo del presente estudio fue evaluar en condiciones de campo diversas substancias químicas convencionales y alternativas para el control del manchado de hojas y cálices en este cultivo.

\section{MATERIALES Y MÉTODOS}

\section{Área de estudio}

Durante los meses de octubre a diciembre de 2014 y 2015 se establecieron bajo condiciones de temporal, dos parcelas experimentales del cultivar "criollo" de jamaica (Hibiscus sabdariffa L.) en los municipios de Ayutla y Tecoanapa, Guerrero, donde se tiene presencia de la enfermedad. De acuerdo con datos de la estación meteorológica 12012, situada a 365 m s. n. m. (metros sobre el nivel del mar), en estos municipios prevalece una temperatura y precipitación promedio anual de $27^{\circ} \mathrm{C}$ y $1,519 \mathrm{~mm}$, respectivamente (16).

\section{Manejo agronómico}

En ambos ciclos de cultivo, en las parcelas experimentales, antes de la siembra se realizaron labores de deshierbe. La siembra se realizó depositando 6 semillas/mata. Aproximadamente 20 días después de la emergencia se realizó el aclareo (tres plantas/mata). Las labores de deshierbe fueron manual a intervalos mensuales y los residuos de las arvenses fueron depositados en el surco. En ambos periodos de evaluaciones no se realizaron labores de fertilización.

\section{Productos evaluados}

Se evaluaron cuatro fungicidas convencionales y cuatro productos biorracionales en las dosis indicadas en la Tabla 1, así como un testigo sin aplicación de productos. Los fungicidas utilizados se seleccionaron en base a los antecedentes de su aplicación para el control de $C$. cassiicola en otros cultivos y su modo de acción FRAC (3) (Fungicide
Resistance Action Committee) (www.frac.info). En ambos ciclos de cultivo se realizaron siete aplicaciones individuales de cada producto, excepto para T4, en él se hicieron dos aplicaciones de benomilo (la primera cuatro semanas antes de la floración y la segunda durante la floración y desarrollo de cálices) y cinco de oxicloruro de cobre entre cada aplicación de benomilo (Tabla 2). En el ciclo 2015, se redujeron los intervalos de aplicaciones de los productos en los estados avanzados de madurez de cálices (Tabla 2), debido a que en el ciclo 2014 se observó que este periodo fue el más susceptible al daño por el patógeno.

\section{Diseño experimental}

El ensayo se estableción bajo un diseño experimental de bloques al azar con cuatro repeticiones. La unidad experimental fue conformada por $4 \operatorname{surcos}$ ( $4 \mathrm{~m}$ de largo x $1 \mathrm{~m}$ de ancho) con seis matas (tres plantas /mata) a una distancia de $0.80 \mathrm{~m}$. Como parcela útil se consideraron sólo las plantas de los dos surcos centrales. Para minimizar la interferencia entre tratamientos se empleó una separación de $2 \mathrm{~m}$ entre bloques.

\section{Variables evaluadas}

Como variables de evaluación se registró la severidad de hojas y cálices con dos escalas logarítmicas diagramáticas diseñadas para cada órgano (12). En cada parcela útil se seleccionó una planta de cada mata y se dividió en tres estratos altitudinales (20) y en cada uno de ellos se evaluaron tres hojas o cálices según la etapa fenológica del cultivo. Se realizaron cinco evaluaciones en hojas a intervalos de 14 días después de la primera aplicación del producto en evaluación y tres en cálices a intervalos de siete días. Se cosecharon las plantas de cada parcela útil /tratamientos y se estimó su peso fresco y seco en gramos.

Durante el periodo de estudio se registró la temperatura y humedad relativa con un Datalogger (HoboWare ${ }^{\circledR}$ ) colocado en el centro de cada parcela experimental a la altura del dosel de las plantas con intervalos de registros de datos cada dos horas.

\section{Análisis estadístico}

Los valores de severidad (valor de cada clase) obtenidos con las escalas diagramáticas (12) se transformaron a grados de infección (\%) con la fórmula descrita por Townsend y Heuberger (18):

Tabla 1. Tratamientos químicos utilizados para el control del manchado de hojas y cálices en jamaica, inducido por Corynespora cassiicola, en Guerrero, México. Ciclos 2014 y 2015.

\begin{tabular}{|c|c|c|c|}
\hline Tratamientos & Código FRAC* & Categoría del producto & Dosis \\
\hline T1. Clorotalonil & Grupo M5 (multi-sitio) & Contacto & $2 \mathrm{~g} / \mathrm{L}$ \\
\hline T 2. Mancozeb & Grupo M3 (multi-sitio) & Contacto & $2 \mathrm{~g} / \mathrm{L}$ \\
\hline T 3. Oxicloruro de cobre & Grupo M1 (multi-sitio) & Contacto & $2 \mathrm{~g} / \mathrm{L}$ \\
\hline \multicolumn{4}{|l|}{ Alternativos } \\
\hline T 5. Acibenzolar-S-methyl & P1(Inductor de defensa de la planta hospedante) & Inductor de resistencia sistémica adquirida. & $100 \mathrm{mg} / \mathrm{L}$ \\
\hline T 6. Dióxido de hidrógeno & No clasificado (NC) & Contacto & $5 \mathrm{~mL} / \mathrm{L}$ \\
\hline T9. Testigo & $\ldots$ & $\ldots$ & $\ldots$ \\
\hline
\end{tabular}

*Fungicide Resistance Action Committee. 
Tabla 2. Programa de aplicaciones de productos químicos, para el control del manchado de hojas y cálices de jamaica, inducido por $C$. cassiicola en condiciones de campo en Ayutla y Tecoanapa, Guerrero. Ciclos 2014 y 2015

\begin{tabular}{|c|c|c|c|c|c|c|}
\hline \multirow[b]{2}{*}{ Localidad } & \multicolumn{3}{|c|}{ Programa de aplicación (2014) } & \multicolumn{3}{|c|}{ Programa de aplicación (2015) } \\
\hline & $\begin{array}{l}\text { Fecha de } \\
\text { aplicación }\end{array}$ & $\begin{array}{l}\text { Intervalo } \\
\text { (días) }\end{array}$ & Etapa fenológica & $\begin{array}{l}\text { Fecha de } \\
\text { aplicación }\end{array}$ & $\begin{array}{l}\text { Intervalo } \\
\text { (días) }\end{array}$ & Etapa fenológica \\
\hline Ayutla & 03 oct & 14 & Desarrollo vegetativo & 20 oct & 14 & Desarrollo vegetativo \\
\hline Tecoanapa & 04 oct & & & 21 oct & & \\
\hline Ayutla & 17 oct & 14 & Desarrollo vegetativo & 04 nov & 14 & Desarrollo vegetativo \\
\hline Tecoanapa & 18 oct & & & 05 nov & & \\
\hline Ayutla & 31 oct & 14 & Desarrollo vegetativo & 18 nov & 14 & Floración y desarrollo de cálices \\
\hline Tecoanapa & 01 nov & & & 19 nov & & \\
\hline Ayutla & 15 nov & 14 & Floración y desarrollo de cálices & 25 nov & 7 & Desarrollo de cálices \\
\hline Tecoanapa & 16 nov & & & 26 nov & & \\
\hline Ayutla & 22 nov & 7 & Desarrollo de cálices & 03 dic & 7 & Desarrollo de cálices \\
\hline Tecoanapa & 23 nov & & & 04 dic & & \\
\hline Ayutla & 30 nov & 7 & Desarrollo de cálices & $10 \mathrm{dic}$ & 7 & Desarrollo de cálices \\
\hline Tecoanapa & $01 \mathrm{dic}$ & & & $11 \mathrm{dic}$ & & \\
\hline Ayutla & 7 dic & 7 & Desarrollo de cálices & 17 dic & 7 & Desarrollo y madurez de cálices \\
\hline Tecoanapa & 8 dic & & & 18 dic & & \\
\hline
\end{tabular}

Tabla 3. Temperaturas y humedades relativas en Ayutla y Tecoanapa, Guerrero. Ciclos 2014 y 2015.

\begin{tabular}{|c|c|c|c|c|c|c|c|c|}
\hline \multirow[b]{2}{*}{ Ciclos de cultivo } & \multirow[b]{2}{*}{ Mes } & \multicolumn{4}{|c|}{$\begin{array}{c}\text { Temperatura } \\
\left({ }^{\circ} \mathrm{C}\right) \\
\end{array}$} & \multicolumn{3}{|l|}{$\begin{array}{c}\text { Humedad } \\
\text { relativa (\%) }\end{array}$} \\
\hline & & Localidad & Promedio & Máxima & Mínima & Promedio & Máxima & Mínima \\
\hline 2014 & Octubre & Tecoanapa & 25.68 & 36.94 & 19.82 & 92.34 & 100.00 & 56.73 \\
\hline$\ll$ & Noviembre & Tecoanapa & 25.56 & 41.57 & 15.56 & 83.66 & 100.00 & 28.91 \\
\hline$«$ & Diciembre & Tecoanapa & 25.01 & 41.52 & 15.31 & 73.78 & 99.91 & 25.09 \\
\hline$«$ & Octubre & Ayutla & 25.35 & 33.47 & 20.71 & 95.67 & 100.00 & 70.66 \\
\hline « & Noviembre & Ayutla & 26.03 & 38.71 & 17.55 & 84.39 & 100.00 & 39.34 \\
\hline$\ll$ & Diciembre & Ayutla & 26.09 & 42.06 & 16.18 & 71.46 & 99.46 & 20.31 \\
\hline 2015 & Octubre & Tecoanapa & 28.24 & 37.65 & 20.88 & 80.32 & 99.21 & 45.67 \\
\hline$\ll$ & Noviembre & Tecoanapa & 25.73 & 35.78 & 20.81 & 84.65 & 99.40 & 49.93 \\
\hline « & Diciembre & Tecoanapa & 26.41 & 47.23 & 17.10 & 78.76 & 97.53 & 23.33 \\
\hline « & Octubre & Ayutla & 27.01 & 35.42 & 21.51 & 94.03 & 100.00 & 68.00 \\
\hline$\ll$ & Noviembre & Ayutla & 27.42 & 41.26 & 20.71 & 86.77 & 100.00 & 43.78 \\
\hline$\ll$ & Diciembre & Ayutla & 26.45 & 40.05 & 19.24 & 81.06 & 100.00 & 36.13 \\
\hline & \multicolumn{2}{|c|}{ Promedio general } & 26.25 & 39.31 & 18.78 & 83.91 & 99.63 & 42.32 \\
\hline
\end{tabular}

Grado de infección $(\%)=\sum_{i=1}^{k}\left(n_{i} \times v_{i}\right) / N \times V$

Donde $v_{i}$ es daño en la clase $i, n_{i}$ es el número en la clase $i, \mathrm{~N}$ es el número total de observaciones, $\mathrm{V}$ es el valor más alto de clases, $k$ es el número de clases.

Los grados de infección de la severidad acumulada en hojas y cálices y el peso fresco y seco por tratamiento fueron sometidos a un análisis de varianza y pruebas de separación de medias con el logaritmo (log) de cada valor (con ajustes de Tukey-Kramer por multiplicidad) con un nivel de significancia de 0.05. Los análisis fueron realizados con el paquete estadístico SAS. Ver. 9.4.

\section{RESULTADOS Y DISCUSIÓN}

El manchado de hojas y cálices de jamaisa se presentó con altos niveles de severidad durante los dos años de evaluación. En los ciclos productivos de 2014 y 2015 para Ayutla y Tecoanapa, las temperaturas promedio oscilaron entre $25.01{ }^{\circ} \mathrm{C}$ y $28.24^{\circ} \mathrm{C}$ con humedades relativas entre $71.46 \%$ y $95.67 \%$ (Tabla 3 ). En base a lo reportado por Teramoto et al. (17), mencionan que las temperaturas entre $23.3{ }^{\circ} \mathrm{C}$ a $29.5^{\circ} \mathrm{C}$ y alta humedad relativa (100\%) (2) son favorables para C. cassiicola, condiciones similares fueron registradas en el presente estudio (Tabla 3 ).

En ambos ciclos de cultivo, en el tratamiento con mancozeb, clorotalonil y benomilo alternado con oxicloruro de cobre se obtuvo 


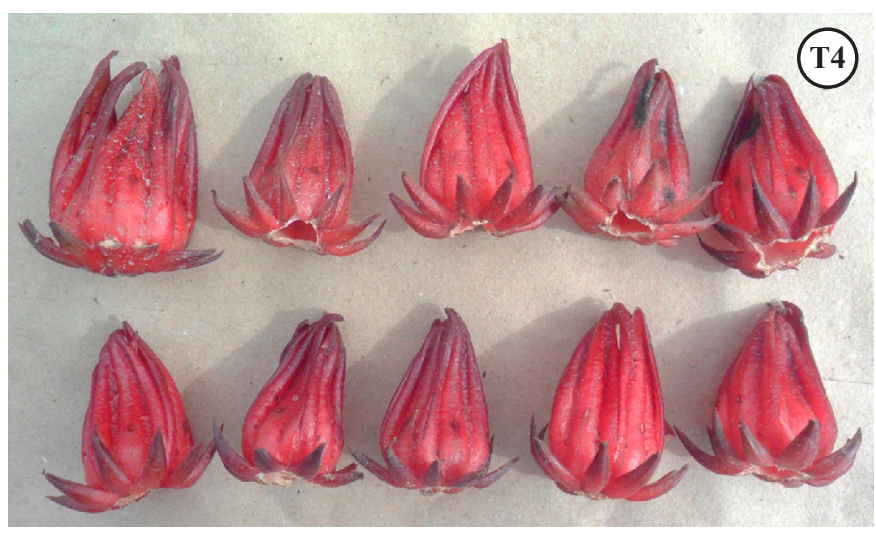

Figura 1. T4; aspecto de los cálices asperjados con Benomilo alternado con oxicloruro de cobre. Ciclo de evaluación en 2014. Tecoanapa, Guerrero, México

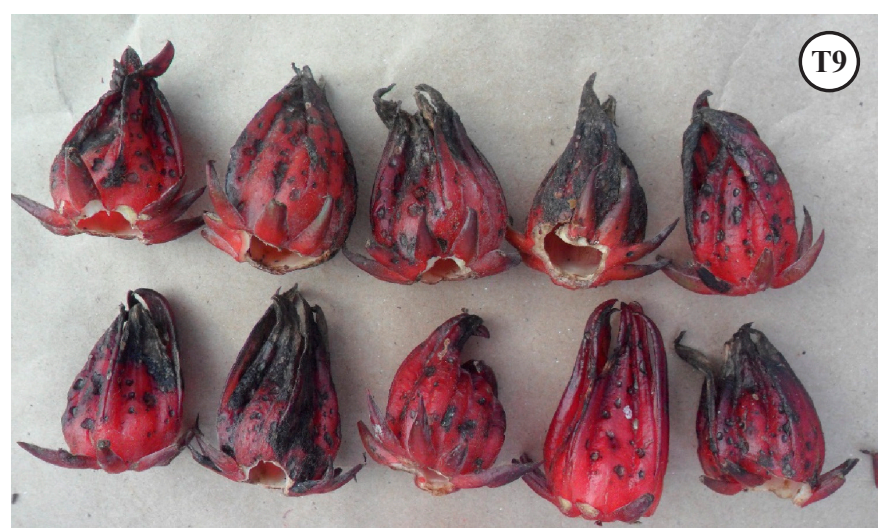

Figura 2. T9 (testigo); aspecto de los cálices sin aplicación de substancia. Ciclo de evaluación en 2014. Tecoanapa, Guerrero, México.

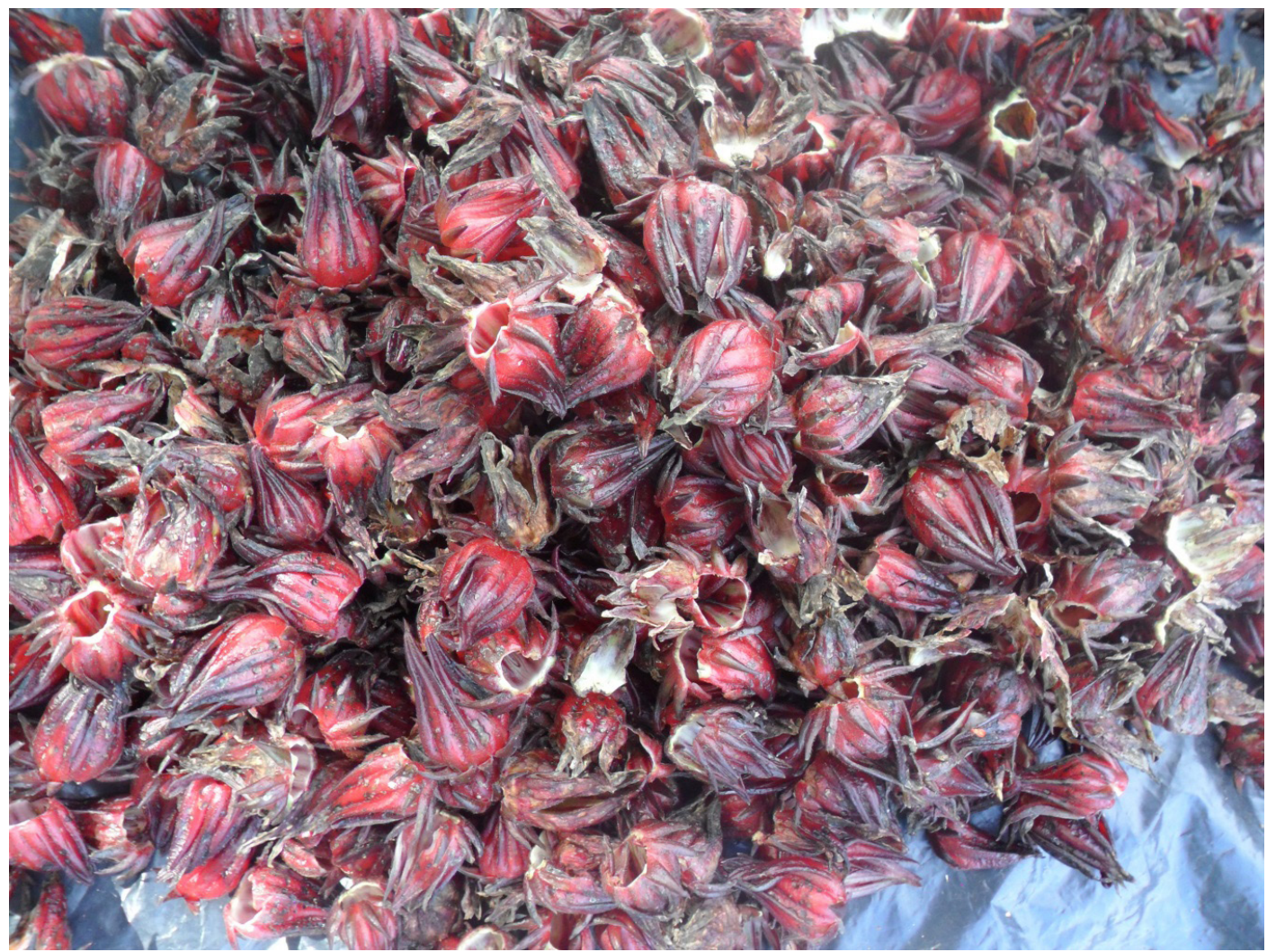

Figura 3. Problemática actual del manchado de la jamaica inducido por el hongo Corynespora cassiicola en Guerrero, México. Aspecto de la cosecha de cálices de jamaica con la enfermedad del manchado en una plantación comercial de jamaica en el municipio de Ayutla, Guerrero. Ciclo 2015. En la figura se puede apreciar una incidencia del 100\% y alto grado de severidad (en algunos cálices $>50 \%$ ). Por el aspecto de los cálices la comercialización se dificulta y en ocasiones se rechaza (el producto es desechado).

un mayor nivel de control de acuerdo con los menores valores de severidad de hojas y cálices obtenidos con respecto al tratamiento testigo (Tabla 4). Al respecto, se ha documentado la efectividad del mancozeb, clorotalonil y benomilo para el control de $C$. cassiicola en otros cultivos, como jitomate y chile dulce con porcentaje de control superiores al $80 \%(13,19)$. Por otra parte, las aplicaciones individuales de oxicloruro de cobre redujeron moderadamente la severidad de hojas y cálices (Tabla 4). Esto coincide con los reportes de Manju et al. (5) en el control de C. cassiicola en Hevea brasiliensis (Willd. ex A. Juss.) Müll. Arg., quienes demostraron la reducción de la enfermedad en más del $50 \%$ con aplicaciones de mancozeb, mientras que con oxicloruro de cobre fue del 40\%. Por su parte, Godoy et al. (4) evaluaron mancozeb para controlar la mancha foliar de soya causada por C. cassiicola, determinaron un porcentaje de control del $48 \%$ al $52 \%$, mientras que con el oxicloruro de cobre fue del $43 \%$.

Mancozeb, clorotalonial y oxicloruro de cobre, son fungicidas de contacto multisitio, con bajo riesgo de inducir resistencia en hongos. El benomilo, actúa de forma sistémica y se ha documentado resistencia a ésta molécula en varias especies de hongos (3).

En esta investigación, la aplicación de benomilo en dos ocasiones 
Tabla 4. Productos químicos utilizados para el control del manchado de hojas y cálices de jamaica inducido por C. cassiicola, en Ayutla y Tecuanapa, Guerrero, México. Ciclos 2014 y 2015

\begin{tabular}{|c|c|c|c|c|c|c|c|c|c|c|c|c|c|c|c|c|}
\hline \multirow{3}{*}{ Tratamiento } & \multicolumn{8}{|c|}{ Severidad en hojas } & & & & \multicolumn{5}{|c|}{ Severidad en cálices } \\
\hline & \multicolumn{3}{|c|}{ Ayutla } & & \multicolumn{3}{|c|}{ Tecoanapa } & \multicolumn{4}{|c|}{ Ayutla } & \multicolumn{5}{|c|}{ Tecoanapa } \\
\hline & $2014^{\S}$ & & 2015 & & 2014 & & 2015 & & 2014 & & 2015 & & 2014 & & 2015 & \\
\hline Mancozeb & 4.82 & $\mathrm{c}$ & 4.76 & $\mathrm{c}$ & 4.73 & e & 4.71 & $\mathrm{~d}$ & 2.41 & $\mathrm{c}$ & 2.34 & $\mathrm{c}$ & 2.14 & $\mathrm{c}$ & 2.10 & $\mathrm{c}$ \\
\hline Oxicloruro de cobre & 5.03 & $\mathrm{bc}$ & 4.99 & $\mathrm{bc}$ & 4.89 & $\mathrm{c}$ & 4.82 & $\mathrm{~cd}$ & 3.69 & $\mathrm{~b}$ & 3.28 & $\mathrm{bc}$ & 3.30 & $\mathrm{bc}$ & 2.84 & $\mathrm{bc}$ \\
\hline Dióxido de hidrógeno & 5.12 & $a b$ & 5.11 & $a b$ & 5.00 & $\mathrm{~b}$ & 4.95 & $\mathrm{~b}$ & 3.95 & ba & 3.85 & $\mathrm{~b}$ & 3.48 & $\mathrm{ba}$ & 3.40 & ba \\
\hline Bicarbonato de potasio & 5.13 & $a b$ & 5.10 & $\mathrm{ab}$ & 5.03 & $\mathrm{~b}$ & 4.98 & $\mathrm{~b}$ & 3.82 & $\mathrm{~b}$ & 3.79 & $\mathrm{~b}$ & 3.34 & ba & 3.28 & ba \\
\hline Aceite del árbol del Té & 5.12 & $a b$ & 5.10 & $\mathrm{ab}$ & 4.98 & $\mathrm{~b}$ & 4.96 & $\mathrm{~b}$ & 4.00 & ba & 3.94 & $\mathrm{~b}$ & 3.48 & $\mathrm{ba}$ & 3.35 & ba \\
\hline Testigo & 5.35 & $\mathrm{a}$ & 5.42 & $\mathrm{a}$ & 5.30 & $\mathrm{a}$ & 5.26 & $\mathrm{a}$ & 4.28 & $\mathrm{a}$ & 5.30 & $\mathrm{a}$ & 3.94 & $\mathrm{a}$ & 4.40 & $\mathrm{a}$ \\
\hline
\end{tabular}

§Año de evaluación. ¥Logaritmo del porcentaje de infección de la severidad. S Separación de medias, Tukey-Kramer, $\alpha=0.05$.

Tabla 5. Productos químicos evaluados para el control del manchado de la jamaica inducido por C. cassiicola y su relación con el peso fresco y seco de cálices en campo, Ayutla y Tecuanapa, Guerrero, México. Ciclos 2014 y 2015.

\begin{tabular}{|c|c|c|c|c|c|c|c|c|}
\hline \multirow[b]{2}{*}{ Tratamientos } & \multicolumn{2}{|c|}{ Ayutla } & \multicolumn{2}{|c|}{ Ayutla } & \multicolumn{2}{|c|}{ Tecoanapa } & \multicolumn{2}{|c|}{$\begin{array}{l}\text { Tecoanapa } \\
2015\end{array}$} \\
\hline & Peso fresco & Peso seco & Peso fresco & Peso seco & Peso fresco & Peso seco & Peso fresco & Peso seco \\
\hline Clorotalonil & $7.95^{*} \mathrm{a}^{\mathrm{S}}$ & $5.89 \mathrm{a}$ & $8.14 \mathrm{a}$ & $6.03 \mathrm{a}$ & $8.17 \mathrm{a}$ & $6.06 \mathrm{a}$ & $7.92 \mathrm{a}$ & $5.78 \mathrm{a}$ \\
\hline Mancozeb & $7.90 \mathrm{a}$ & $5.82 \mathrm{a}$ & $8.13 \mathrm{a}$ & $6.02 \mathrm{a}$ & $8.15 \mathrm{a}$ & $6.05 \mathrm{a}$ & $7.95 \mathrm{a}$ & $5.78 \mathrm{a}$ \\
\hline Benomilo + Oxicloruro de cobre & $7.86 \mathrm{a}$ & $5.77 \mathrm{a}$ & $8.08 \mathrm{a}$ & $6.01 \mathrm{a}$ & $8.13 \mathrm{a}$ & $6.00 \mathrm{a}$ & $7.79 \mathrm{a}$ & $5.72 \mathrm{a}$ \\
\hline Acibenzolar-S-Methyl & $7.36 \mathrm{ab}$ & $5.29 \mathrm{abc}$ & $7.63 \mathrm{bc}$ & $5.81 \mathrm{bc}$ & $7.90 \mathrm{ab}$ & $5.81 \mathrm{ab}$ & $7.43 \mathrm{ab}$ & $5.35 \mathrm{bc}$ \\
\hline Dióxido de hidrógeno & $7.24 \mathrm{~b}$ & $5.18 \mathrm{bc}$ & $7.60 \mathrm{bc}$ & $5.64 \mathrm{bd}$ & $7.74 \mathrm{~b}$ & $5.65 \mathrm{bc}$ & $7.34 \mathrm{ab}$ & $5.26 \mathrm{bcd}$ \\
\hline Bicarbonato de potasio & $7.41 \mathrm{ab}$ & $5.34 \mathrm{abc}$ & $7.62 \mathrm{bc}$ & $5.80 \mathrm{bd}$ & $7.90 \mathrm{ab}$ & $5.81 \mathrm{abc}$ & $7.49 \mathrm{ab}$ & $5.29 \mathrm{bcd}$ \\
\hline
\end{tabular}

§Año de evaluación. ¥Logaritmo del peso. S Separación de medias, Tukey-Kramer, $\alpha=0.05$.

alternado con oxicloruro de cobre resultó en menor severidad en hojas y cálices (Tabla 4). La FRAC (3), sugiere reducir el número de aplicaciones de benomilo durante el ciclo de cultivo para evitar el desarrollo de resistencia, por lo anterior en el presente estudio se realizaron dos aplicaciones de benomilo alternado con oxicloruro de cobre. En Nigeria, el benomilo ha controlado en más del 40\% y $60 \%$ la incidencia y severidad, respectivamente, causada por Coniella musaiensis var. hibisci B. Sutton, patógeno de la jamaica (8).

Los productos alternativos (Tabla 1) en general promovieron menor severidad comparados con la registrada en el tratamiento testigo (Tabla 4); resultados similares fueron reportados por Becktell et al. (1), quienes evaluaron al bicarbonato de potasio, dióxido de hidrógeno y otras substancias para el control de Phytophthora infestans (Mont.) de Bary, en petunia (Petunia $\mathbf{x}$ hybrida Hort. ex E. Vilm.), ellos determinaron que estas substancias fueron menos efectivas ya que la severidad de la enfermedad fue de alrededor del 15\%; mientras que en los tratamientos con dimethomorph/mancozeb y fosetyl-aluminio fue menor al $4 \%$. No obstante, debido a que el bicarbonato de potasio, dióxido de hidrógeno, acibenzolar-s-methyl y aceite del árbol del té (Melaleuca alternifolia
Cheel), tienen menor impacto ambiental y redujeron moderadamente la severidad de la enfermedad del manchado, pueden resultar útiles en un programa de manejo si se combinan con algún fungicida convencional. Al respecto Marine et al. (6), evaluaron al dióxido de hidrógeno y otros productos biorracionales con rotaciones de fungicidas a base de cobre para el control de Pseudoperonospora cubensis (Berk. \& M.A. Curtis) Rostovzev y Podosphaera xanthii (Castagne) U. Braun \& Shishkoff, ellos determinaron que este sistema de manejo es una opción viable para la producción orgánica de calabacita y otras cucurbitáceas, ya que permite reducir el número de aplicaciones de fungicidas a base de cobre y lograr un buen manejo de la enfermedad.

El acibenzolar-s-methyl mostró una tendencia en promover menor severidad en hojas y cálices con respecto a los productos alternativos (Tabla 4). Obanor et al. (9) observaron que esta substancia tuvo una efectividad del $48 \%$ a $68 \%$ mientras que con el sulfato de cobre esta fue del $94 \%$ en el control de Spilocaea oleagina (Castagne) S. Hughes, causante de la mancha del olivo (Olea europea L.), a pesar de lo cual sugirieron el uso del primero en programas de manejo de esta enfermedad por ser un producto de bajo impacto ambiental. No 
se encontrón reportes de los intervalos necesarios para la inducción de resistencia contra el manchado de la jamaica. Sin embargo, diversos reportes indican que los periodos para inducir resistencia varían de 1 a 7 días, dicha variación está condicionada principalmente por el cultivo y del tipo de patógeno (10).

Por otra parte, el mayor peso fresco y seco de cálices fue en aquellos tratamientos que mostraron los menores niveles de severidad (clorotalonil, mancozeb y benomilo alternado con oxicloruro de cobre) (Tabla 4 y 5). Estos resultados fueron similares a los reportados por Nejo et al. (8) quienes aplicaron benomilo en jamaica contra C. musaiensis $\mathrm{y}$ demostraron que las plantas tratadas con este fungicida presentaron mayor peso de cálices.

Los resultados de la presente investigación permiten concluir que las plantas de jamaica tratadas con mancozeb, clorotalonil y benomilo alternado con oxicloruro de cobre promovieron la menor severidad de manchado de hojas (4.92 a 4.71) y cálices (2.10 a 2.41) inducida por Corynespora cassiicola y registraron el mayor peso fresco (7.79 a 8.17) y seco (5.72 a 6.06) de cálices respectos a los productos alternativos $\mathrm{y}$ tratamiento testigo.

\section{AGRADECIMIENTOS}

El primer autor agradece al CONACYT por la beca otorgada para realizar estudios de doctorado. Al Colegio de Postgraduados y al proyecto "163972" del Fondo Sectorial SAGARPA-CONACYT por haber contribuido al financiamiento de la presente investigación.

\section{REFERENCIAS}

1. Becktell, M.C.; Daughtrey M.L.; Fry W.E. Epidemiology and management of petunia and tomato late blight in the greenhouse. Plant Disease, Saint Paul, v.89, p.1000-1008, 2005.

2. Fernando, T.H.P.S.; Jayasinghe, C.K.; Wijesundera, R.L.C.; Siriwardane, D. Some factors affecting in vitro production, germination and viability of conidia of Corynespora cassiicola from Hevea brasiliensis. Journal of the National Science Foundation of Sri Lanka, Colombo, v. 40, p. 241-249, 2012.

3. FRAC. Fungicide Resistance Action Committee. List of Plant pathogenic organisms resistant to disease control agents. Crop Life, Brussels. 2016. Belgium. Disponible en: http://www.frac.info/. Consultado: 12/10/2016.

4. Godoy, C.V.; Utiamada C.M.; Meyer, M.C.; Campos, H. D.; Pimenta B.C.; Miguel-Wruck, S. D.; Borges E. P. Eficiência de fungicidas para o controle da mancha-alvo, Corynespora cassiicola, na Safra 2015/16: resultados sumarizados dos ensaios cooperativos. Embrapa Soja, Londrina. Circular Técnica 120, p.1-6, 2016.

5. Manju, M.J.; Jacob, C.K.; Idicula S.P., Vinod, K.K. Corynespora leaf fall disease management in Hevea using oil-dispersible and dust fungicide formulations. Indian Journal of Natural Rubber Research, Kottayam, v.15 n.1, p. 44-48, 2002.
6. Marine, S.C.; Newark, M.J.; Korir, C.R.; Everts, L.K. Evaluation of Rotational Biopesticide Programs for Disease Management in Organic Cucurbit Production. Plant Disease, Saint Paul, v.100 n.11, p.2226-2233, 2016.

7. Marrone, P.G. Barriers to adoption of biological control agents and biological pesticides. In: Radcliffe, E.B.; Hutchison, W.D.; Cancelado, R.E. Integrated Pest Management: Concepts, Tactics, Strategies and Case Studies. Radcliffe, eds. Cambridge University Press, Cambridge, 2009. Cap. 13.p. 163-178.

8. Nejo, O.J. Adeotia, A.Y.A.; Popoolaa, A.R.; Kehindeb, I.A. Effect of foliar application of fungicides on incidence and severity of leaf spot disease of roselle induced by Coniella musaiaensis B. Sutton var. hibisci. Moor Journal of Agricultural Research, Ibadan, n.8, p.263-67, 2007.

9. Obanor, O.F.; Walter, M.; Jones, E.E.; Jaspers, M.V. Efficacy of systemic acquired resistance inducers in olive leaf spot management. Australasian Plant Pathology, Murdoch, n.42, p.163-168, 2013.

10. Oliveira, A.A.R.; W. Nishijima, W. Induction of Resistance to Papaya Black Spot Elicited by Acibenzolar-S-Methyl. Plant Pathology Journal, Deira, v. 13, p. 120-124, 2014.

11. Ortega-Acosta, S.Á.; Hernández-Morales, J.; Ochoa-Martínez, D.L.; Ayala -Escobar, V. First report of Corynespora cassiicola causing leaf and calyx spot on roselle in Mexico. Plant Disease, Saint Paul, v.99, n.7, p.1041, 2015.

12. Ortega-Acosta, S.Á.; Velasco-Criz, C.; Ochoa-Martínez, D.L.; Hernández -Ruiz, J.; Hernández-Morales, J. Escalas logarítmicas diagramáticas para evaluar la severidad del manchado de hojas y cálices de jamaica. Revista Mexicana de Fitopatología, Texcoco, v.34: p.270-285, 2016.

13. Pernezny, K.; Stofella, P.; Collins, J.; Carroll, A.; Beaney, A. Control of target spot of tomato with fungicides, systemic acquired resistance activators, and a biocontrol agent. Plant Protection Science, Prague, v.38, n. 3, p. $81-88,2002$.

14. Sarma, B.K.; Ameer Basha, S.; Singh, D.P.; Singh, U.P. Use of non-conventional chemicals as an alternative approach to protect chickpea (Cicer arietinum) from Sclerotinia stem rot. Crop Protection, Queensland, v. 26, p.1042-1048, 2007.

15. SIAP. Servicio de Información Agroalimentaria y Pesquera. México. 2017. Disponible en: http://www.siap.gob.mx/index.php?option=com_wrapper\&view=wrapper\&Itemid=351. Consultado: 11 de mayo 2017.

16. SMN. Servicio Meteorológico Nacional. Comisión Nacional del Agua Normales climatológicas, Periodo (1981- 2010). 2017. México. Disponible en: http://smn.cna.gob.mx/index.php?option $=$ com_content\&view=article \&id=182: guerrero\&catid=14: normal es-por-estacion. Consultado: 09 de agosto 2017.

17. Teramoto, A.; Parisi, C.M.C.; Cunha, G.M.; Caracterização fisiológica de isolados de Corynespora cassiicola. Tropical Plant Pathology, Brasilia, v.38, n.4, p.313-322, 2013.

18. Townsend, G.R.; Heuberger, J.W. Methods for estimating losses caused by diseases in fungicides experiments. Plant Disease Reporter, Washington, v.27, p.340-343, 1943.

19. Tun-Suárez, J.M.; Castillo-Peraza, M.E.; Cristobal-Alejo, J.; Moreno, L.L. Etiología de la mancha foliar del chile dulce (Capsicum annuum $\mathbf{L}$.) y su control in vitro en Yucatán, México. Fitosanidad, La Habana, v.15, n.1, p.05-10, 2011.

20. Villanueva-Couoh, E.; Sánchez-Briceño., M.Á.; Cristóbal-Alejo, J.; Ruiz-Sánchez, E.; Tun-Suarez, J.M. Diagnóstico y alternativas de manejo químico del tizón foliar (Alternaria chrysanthemi Simmons y Crosier) del crisantemo (Chrysanthemum morifolium Ramat.) Kitamura en Yucatán, México. Revista Mexicana de Fitopatología, Texcoco, v.23, p.49-56, 2005. 